\title{
Enhancement of digital chest images using a modified Sobel edge detection algorithm
}

\author{
Archana J. N. ${ }^{1}$, Aishwarya P. ${ }^{2}$, Hanson Joseph ${ }^{3}$ \\ ${ }^{1}$ Bharathiar University, Coimbatore, India \\ ${ }^{2}$ Department of Computer Science and Engineering, Atria Institute of Technology, Bangalore, India \\ ${ }^{3}$ National Institute of Technology Calicut, Kozhikode, India
}

\begin{tabular}{l} 
Article Info \\
\hline Article history: \\
Received Aug 13, 2021 \\
Revised Oct 19, 2021 \\
Accepted Oct 26, 2021 \\
\hline
\end{tabular}

\section{Keywords:}

Direction template

Edge detection

Image fusion

Modified Sobel 12D algorithm

\begin{abstract}
Computed tomography (CT) images are an essential factor in the diagnosing procedure for various diseases affecting the internal organs. Edge detection can be used for the appropriate enhancement of the lung CT scan images for the diagnosis of the various interstitial lung diseases (ILD). In order to solve the issues of edge detection provided by the traditional Sobel operator, the paper proposes a Sobel 12D edge detection algorithm which uses the additional direction templates for the better identification of the edge details. First, the vertical and horizontal directions available in the traditional Sobel operator are extended to few more directions (a total of 12 directions) which enhances the edge extraction ability. Next part, compute the edge detected image using the Sobel 12D, Laplace, Prewitt, Robert's Cross and Scharr operators for edge detection separately. It is followed by image fusion method which optimizes the edge detection by combining the edge detected images obtained using the Sobel 12D approach and the Laplace operator. The experimental results shows that the proposed algorithms generates a better detection of the edges than the other edge detection operators.
\end{abstract}

This is an open access article under the CC BY-SA license.

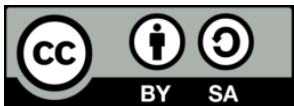

\section{Corresponding Author:}

Archana J. N.

Bharathiar University

Tamil Nadu 641046, Coimbatore, India

Email: archanajn.cse@gmail.com

\section{INTRODUCTION}

There is a wide range of application of the digital images in various areas specifically in medical field. Digital images are used in the advanced imaging techniques such as magnetic resonance imaging (MRI), computed tomography (CT), ultrasound and X-rays [1]. In these techniques there is a possibility of the interference of noise in the images generated [2]. Hence in order to eliminate these noises there is a necessity of improving the clarity of the images generated. The process of identification of the smaller units in the images and enhancing the features of the image is image enhancement. Image enhancement improves the details of the image which results in better appearance of the image. There are many enhancement techniques available such as image sharpening, edge detection and image smoothing. This process has much significance in medical image diagnosis. As a result of the enhancement of medical images of human organs the physician can diagnose the internal features for the identification of various diseases.

There is a high increase in the cases of lungs related diseases due to the issues such as pollution, life style change and family history. Many of the lung related diseases if diagnosed at the initial stage can be recovered. The perfect diagnosis of these diseases will be mainly dependent on the quality of the images generated using the various scanning technique [3]. We discuss in this paper about the diagnosis of idiopathic 
pulmonary fibrosis (IPF). IPF is a type of chronic interstitial lung diseases (ILD) which affects the internal parts of lungs. The early diagnosis of the disease can help the physician understand the stages of the disease. Based on the appropriate identification from the CT scan lung images, immediate medication can be suggested. The requirements and the constraints to be considered for the image-based diagnosis of various categories of ILD which uses only the support of the CT scan images D [4]. There are many methods identified and used in the detection of the various types of Interstitial Lung Diseases such as lung cancer, IPF [3], [5].

IPF results in gradual fibrosing and mostly shows cases of pneumonia which affects the interstitium of the lungs. In most of the cases reported it is diagnosed in the older adults with symptoms of malfunctioning of the lungs. There are a few cases of IPF such as Intralobular interstitial thickening (IIT), IPF thickening, parenchymal bands (PB) and honeycombing (HC). In most of the cases there are many details available in the images which needs to be identified specifically along the edge region. Hence identification of an approach which focuses on the edges appears to be very important.

Edges contains the most valuable information in an image. Due to this, the retrieval of edge content in an image is a very important part in image enhancement. The edge information retrieved should be of very good quality. Along with that the complete knowledge about the properties of the image is obtained. So as a part of improving the quality in an image there is a necessity of identification of the edges. Hence the process of identification of the edges in an image is referred as edge detection [6]. Study on the performance of the various edge detectors such as Sobel, Prewitt, Robert, Laplacian, Canny operators discussed [7], [8]. Also, it highlights the significance of the frequency components of the edge detection operators. Edge preservation is considered as one of the basic step in edge detection. Particularly in medical image analysis, where the identification of diseases by the physician is done using the various imaging techniques such as CT scan and X-rays. Filters suggested as a part of denoising identifies and removes the unwanted noise in these images [9], [10].

\section{RELATED WORKS}

Edge detection is applied for the investigation of the image details of medical, satellite, forensics and vehicular images traffic maintenance. There are few techniques used in the diagnosis of interstitial lung diseases available in literature. For example, Wong et al. [11] suggest methods to diagnose and classify the different types of ILD. Several updated illustrations of the identification of ILD using various techniques in CT scan images [3]. The identification of the advancement of the stages of IPF and the diagnosis based on the symptoms using the various scanning techniques illustrated [12], [13].

The extraction of specific parts in an image are performed using the various enhancement techniques. There are many research works based on the application of edge detection operators such as Laplacian, Sobel, Canny, Prewit [14], [15]. Similarly [16] focuses on Haar wavelets, Sobel, Gabor and Laplacian filters. Edge detection using a threshold parameter provides better enhancement results on images [16]. There is more focus given in object detection as patterns using the Laplacian operator together with the convolution approach [17]. Among the various operators available for edge detection Laplacian operators also provides good results. It is applied for pedestrian detection for driving assistance systems of cars and other vehicles [18]. Literature on various categories of edge detection algorithms such as ISEF, Canny, Marr-Hildreth, Sobel, Kirsch, Lapla1 and Lapla2 shown with classification based on the pros and cons discussed [19].

The edge detection operators are categorized based on two methods such as the gradient and Laplacian. Sobel, Laplace and Canny are few categories of gradient based filters used for edge detection [20]. The modified Sobel edge detection approaches preserves the sharp thin and noiseless edges [21]. White Gaussian noise is eliminated using an approach which combines the Sobel edge detection with soft threshold wavelet noise removal scheme [22]. Among the various edge detection algorithms available, the concepts of gradient orientation and the direction templates are mostly applied in medical imaging. Sobel operators performs the gradient calculation in the horizontal and vertical directions [23]. The identification of the edges in the other directions are performed as a result of the extension of the direction templates to other directions. There is more work available in the literature based on the direction template extension performed on radar remote sensing with four directions [24] grayscale face images two directions [25] hardware applications [26], [27] provides better accuracy and edge perseverance with continuity of the edges. The results generated using these algorithms are found to be of less significance in noisy images. Identification of exceptional objects as well as growths in human parts were diagnosed using the images generated. There are various regions in these images which needs to be highlighted. A filter generated using the Markov approach along with the Laplacian operation is applied here to detect the medical image's edges [28].

The method of the combination of the operator with the current technique called image fusion provides better enhancement results. The image fusion approach combines the Sobel operator with other operators like Canny and Laplacian of Gaussian (LoG) operator and solves most of the issues with the normal Sobel operators. The smoothing extension of the direction templates used helps in the edge 
extraction. The overall results generated using the approach increases the possibilities of better edge detection [29].

\subsection{Traditional Sobel operator}

Sobel filter is a convolution filter used for edge detection in images. The derivative of the image is calculated for edge detection. The high magnitude estimated region in an image using the derivatives are considered as the edges. There are two $3 \times 3$ filters applied in the approach assigned for the horizontal and vertical direction edges identification. As a result of the application of the filters there are two gradient images generated.

The edge detected image generated is shown in (1),

$$
\mathrm{G}=|\mathrm{Gx}|+|\mathrm{Gy}|
$$

where Gx and Gy represents the gradient in $\mathbf{x}$ and $\mathbf{y}$ direction respectively. The convolution template of the original Sobel Filters or mask can be represented as shown in Figure 1.

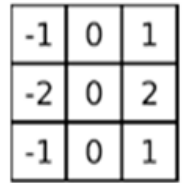

(a)

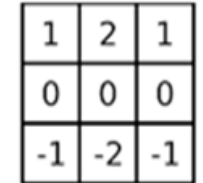

(b)

Figure 1. Sobel filters: (a) horizontal Sobel filter and (b) vertical Sobel filter

The traditional Sobel operators performs the gradient calculation along the horizontal and vertical directions only. Due to which the sufficient information about edges apart from these horizontal and the vertical directions cannot be identified. The measurement of the gradients in different other directions helps in the identification of the edges accurately from the image given. The application of this proposed approach of Sobel edge detection succeeds in the detection better than the conventional methods.

\section{PROPOSED EDGE DETECTION APPROACH}

\subsection{Proposed Sobel 12D edge detection approach}

In the approach used here, the gradient information of each successive $30^{\circ}$ from $0^{\circ}$ to $330^{\circ}$ is calculated. Let the gradients be assumed as $\mathrm{G}_{0}, \mathrm{G}_{30}, \mathrm{G}_{60}, \ldots, \mathrm{G}_{330}$. The final edge detected image, $\mathrm{G}_{\text {final }}$ is obtained as pixel-by-pixel comparison of all the gradient images. For a given position (i, j), the $(i, j)^{\text {th }}$ pixel value across all the gradient images are compared; and the maximum pixel value is assigned to the (i, j) ${ }^{\text {th }}$ position of the final output edge image.

$$
G_{\text {final }}(i, j)=\max \left(G_{0}(i, j), G_{30}(i, j), G_{60}(i, j), \ldots \ldots, G_{330}(i, j)\right)
$$

Or a $3 \times 3$ section of an image, the gradient can be estimated with respect to the $\mathrm{x}$ and $\mathrm{y}$ axis as shown in the (2),

$$
\begin{aligned}
& G=(2(f-d)+(c+i-a-g)) \hat{\imath}+(2(b-h)+(a+c-g-i)) \hat{\jmath} \\
& G=M \hat{\imath}+N \hat{\jmath}
\end{aligned}
$$

where $\mathrm{G}$ represents the gradient and the pixel values are a, b, c, d, e, f, g, h, and i.

The implementation of the traditional Sobel operators are simple and fast. There are thicker edges identified with less accuracy due to the usage of only two direction templates such as horizontal and vertical templates [30]. So, in order to improve the edge features of the image to be diagnosed the Sobel operator direction templates can be extended by including few more directions. In addition to directions 0 and 90 there are 10 more direction templates included such as $30^{\circ}, 60^{\circ}, 120^{\circ}, 150^{\circ}, 180^{\circ}, 210^{\circ}, 240^{\circ}, 270^{\circ}, 300^{\circ}$ and $330^{\circ}$. Figure 2 shows the twelve direction templates. Each of the twelve direction templates are used for the operation of convolution in a pending image pixel by pixel from left to right and from top to bottom; thereby resulting in twelve convoluted images. These images are used to build the final edge image. Each pixel of the final edge image is obtained by taking the maximum value among corresponding pixels of these 12 images. 


\begin{tabular}{|c|c|c|}
\hline-1 & 0 & +1 \\
\hline-2 & 0 & +2 \\
\hline-1 & 0 & +1 \\
\hline
\end{tabular}

(a)

\begin{tabular}{|c|c|c|}
\hline+1 & +2 & +1 \\
\hline 0 & 0 & 0 \\
\hline-1 & -2 & -1 \\
\hline
\end{tabular}

(d)

\begin{tabular}{|c|c|c|}
\hline+1 & 0 & -1 \\
\hline+2 & 0 & -2 \\
\hline+1 & 0 & -1 \\
\hline
\end{tabular}

(g)

\begin{tabular}{|c|c|c|}
\hline-1 & -2 & -1 \\
\hline 0 & 0 & 0 \\
\hline+1 & +2 & +1 \\
\hline
\end{tabular}

(j)

\begin{tabular}{|c|c|c|}
\hline $\begin{array}{l}+1 / 2 \\
-\sqrt{ } 3 / 2\end{array}$ & +1 & $\begin{array}{l}+1 / 2 \\
+\sqrt{ } 3 / 2\end{array}$ \\
\hline$\cdot \sqrt{3}$ & 0 & $+\sqrt{3}$ \\
\hline $\begin{array}{l}-1 / 2 \\
-\sqrt{ } 3 / 2\end{array}$ & -1 & $\begin{array}{l}-1 / 2 \\
+\sqrt{3} / 2\end{array}$ \\
\hline
\end{tabular}

(b)

\begin{tabular}{|c|c|c|}
\hline $\begin{array}{l}+1 / 2 \\
+\sqrt{ } 3 / 2\end{array}$ & $+\sqrt{3}$ & $\begin{array}{l}-1 / 2 \\
+\sqrt{3} / 2\end{array}$ \\
\hline+1 & 0 & -1 \\
\hline $\begin{array}{l}+1 / 2 \\
-\sqrt{3} 3 / 2\end{array}$ & $\cdot \sqrt{3}$ & $\begin{array}{l}-1 / 2 \\
-\sqrt{ } 3 / 2\end{array}$ \\
\hline
\end{tabular}

(e)

\begin{tabular}{|l|c|c|}
\hline $\begin{array}{l}-1 / 2 \\
+\sqrt{3} / 2\end{array}$ & -1 & $\begin{array}{l}-1 / 2 \\
-\sqrt{3} / 2\end{array}$ \\
\hline$+\sqrt{3}$ & 0 & $-\sqrt{ } 3$ \\
\hline$+1 / 2$ & +1 & $\begin{array}{l}+1 / 2 \\
-\sqrt{3} / 2\end{array}$ \\
\hline
\end{tabular}

(h)

\begin{tabular}{|c|c|c|}
\hline $\begin{array}{l}-1 / 2 \\
-\sqrt{3} / 2\end{array}$ & $-\sqrt{3}$ & $\begin{array}{c}+1 / 2 \\
-\sqrt{3} / 2\end{array}$ \\
\hline-1 & 0 & +1 \\
\hline $\begin{array}{l}-1 / 2 \\
+\sqrt{3} / 2\end{array}$ & $+\sqrt{ } 3$ & $\begin{array}{l}+1 / 2 \\
+\sqrt{ } 3 / 2\end{array}$ \\
\hline
\end{tabular}

(k)

\begin{tabular}{|c|c|c|}
\hline $\begin{array}{l}-1 / 2 \\
+\sqrt{ } 3 / 2\end{array}$ & $+\sqrt{ } 3$ & $\begin{array}{l}+1 / 2 \\
+\sqrt{ } 3 / 2\end{array}$ \\
\hline-1 & 0 & +1 \\
\hline $\begin{array}{l}-1 / 2 \\
-\sqrt{3} / 2\end{array}$ & $-\sqrt{3}$ & $\begin{array}{l}+1 / 2 \\
-\sqrt{3} / 2\end{array}$ \\
\hline
\end{tabular}

(c)

\begin{tabular}{|l|c|c|}
\hline $\begin{array}{l}+1 / 2 \\
+\sqrt{3} / 2\end{array}$ & +1 & $\begin{array}{l}+1 / 2 \\
-\sqrt{3} / 2\end{array}$ \\
\hline$+\sqrt{ } 3$ & 0 & $-\sqrt{3}$ \\
\hline $\begin{array}{l}-1 / 2 \\
+\sqrt{ } 3 / 2\end{array}$ & -1 & $\begin{array}{l}-1 / 2 \\
-\sqrt{3} / 2\end{array}$ \\
\hline
\end{tabular}

(f)

\begin{tabular}{|c|c|c|}
\hline $\begin{array}{l}+1 / 2 \\
-\sqrt{3} / 2\end{array}$ & $-\sqrt{3}$ & $\begin{array}{l}-1 / 2 \\
-\sqrt{3} / 2\end{array}$ \\
\hline+1 & 0 & -1 \\
\hline $\begin{array}{l}+1 / 2 \\
+\sqrt{ } 3 / 2\end{array}$ & $+\sqrt{3}$ & $\begin{array}{l}-1 / 2 \\
+\sqrt{ } 3 / 2\end{array}$ \\
\hline
\end{tabular}

(i)

\begin{tabular}{|c|c|c|}
\hline $\begin{array}{l}-1 / 2 \\
-\sqrt{3} / 2\end{array}$ & -1 & $\begin{array}{l}-1 / 2 \\
+\sqrt{ } / 2\end{array}$ \\
\hline$-\sqrt{3}$ & 0 & $+\sqrt{ } 3$ \\
\hline$+1 / 2$ & +1 & $\begin{array}{l}+1 / 2 \\
+\sqrt{ } 3 / 2\end{array}$ \\
\hline
\end{tabular}

(I)

Figure 2. Direction templates, (a) Sobel $0^{\circ}$ filter, (b) Sobel $30^{\circ}$ filter, (c) Sobel $60^{\circ}$ filter, (d) Sobel $90^{\circ}$ filter, (e) Sobel $120^{\circ}$ filter, (f) Sobel $150^{\circ}$ filter, (g) Sobel $180^{\circ}$ filter, (h) Sobel $210^{\circ}$ filter, (i) Sobel $240^{\circ}$ filter,

(j) Sobel $270^{\circ}$ filter, (k) Sobel $300^{\circ}$ filter, (l) Sobel $330^{\circ}$ filter

\subsection{Sobel 12D approach}

Based on the various factors estimated in the previous section the step wise description of the Sobel 12D edge detection approach is described. Convert the input lung image into Grayscale image. Extend the direction of the templates to 10 different directions apart from the horizontal and vertical directions. Convolving the lung image with the 12 templates separately to generate 12 convolved images. The gradient value is calculated as a new gray value by using the convolution values. Find edges using the Sobel convolution filters. Combine the resulting edge images using max. Generate the edge detected output image. Figure 3 illustrates the Sobel 12D approach using the phases such as the calculation of the convolution filters followed by the edge identification. At the last phase the combination using the maximum corresponding pixel results in the final edge detected output image.

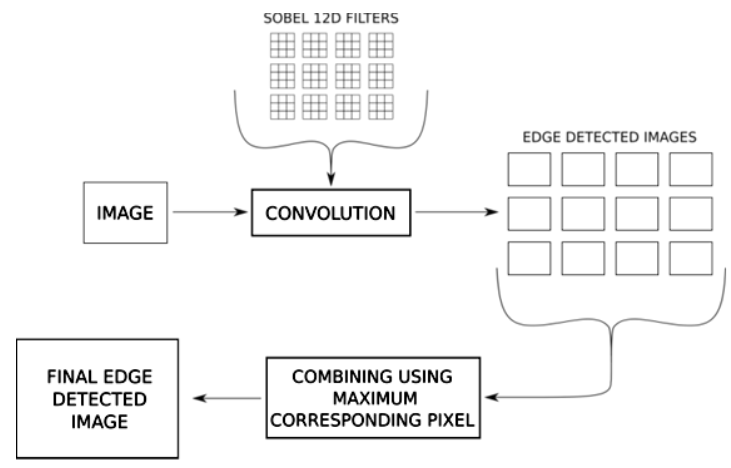

Figure 3. Block diagram of Sobel 12D algorithm 


\subsection{Image fusion approach}

The various operators used in edge detection provides and considers the different issues in edges to be identified. Each operator contributes in its own pattern of generating the edge preserved and smoothness output image. While some operators are better at identifying the thin edges in different orientations, there are a few which could be suggested to discard or eliminate the random noise. Hence there is a possibility of obtaining a better edge preserved image as a result of the combination of the output images generated using the gradient operators of edge detection. Th image fusion approach combines the image obtained from the Sobel 12D approach and the Laplacian operator. Laplacian operator is suggested as it considers the edge details with continuity in maintaining the edge preservation index. Here a weighted average of the edge preserved output images obtained using the gradient filters are used for better results.

Sobel 12D and Laplace filters are applied on the input image to obtain the output images represented as $\mathrm{S}$ and $\mathrm{L}$ respectively. The weights assumed for the operators are 1 for both the filters. The final edge preserved image is obtained as a result of the combination of the weighted average of the Sobel 12D edge image and Laplace edge image as illustrated in (3).

$$
E=(1 \times S+1 \times L) /(1+1)
$$

Here Sobel 12D operators, Laplace filters and the edge preserved images are denoted as S, L and E respectively. As a part of detection of the edges in the lung images a fusion method is proposed which combines the Sobel 12D approach along with the Laplacian operator. The quality of the edge detected lung image is very much better compared to the other results generated. The various operators used in edge detection provides and considers the different issues in edges to be identified.

The enhancement of the images is performed by taking a difference between the edge detected image and the original input image. Here, the edge image coefficients are scaled by a factor value of 0.5 before taking the difference as a part to limit the sharpening effects. Due to which there will be selective darkening of the edges in the resulting image, which increases the contrast of the edge regions with respect to other regions. Along with this the boundaries of the other regions in the image becomes well defined and results in the sharpening of the image. The image obtained using this step is the final enhanced output image with good clarity. Hence the diagnosis becomes easier for the physician.

\section{EXPERIMENTAL RESULTS AND OBSERVATIONS}

MATLAB R2019a is used in the implementation of the algorithm. IPF lung images are used in the experiment. There are few cases of IPF such as Intralobular Interstitial thickening, IPF thickening parenchymal bands and honeycombing. In most of the cases there are many details available in the images which needs to be identified specifically along the edge region. Hence identification of an approach which focuses on the edges appears to be very important.

The computational experiments are performed using the variety of filters such as Sobel 12D, Prewitt, Robert's Cross, Laplace, Scharr. In addition to that the combination or the fusion of the weighted averages of the Laplace filter with the proposed Sobel 12D algorithm is performed. Experiments were performed using a few critical cases of pulmonary images. The final edge detected image generated as a result of the application of a few of the existing edge detection operators (Prewitt, Robert's Cross, Scharr), Laplacian operator and the 12D Sobel edge detection operator are shown using the result generated. Based on the output image generated on all the cases the comparison is performed to evaluate the analysis. The input lung images used in the work for edge detection includes the cases of Intralobular interstitial thickening, honeycombing, IPF thickening. There are also a few traditional edge detection operators used to enhance the pulmonary images. It can be experimentally shown that these operators have distinct advantages in the detection of edges of an image.

Figure 4 shows the enhanced results of "intralobular interstitial thickening image" where Figure 4(a) is the original Input image, in an effort to illustrate the working of the proposed algorithms there are Figures 4(b)-(g) included in the illustration. Figure 4(b) shows the output obtained as a result of the Sobel $12 \mathrm{D}$ algorithm. Even though the image generated using the proposed algorithm identifies the edges there were few noise distracted parts identified in the image. The results generated for Prewitt, Robert's Cross, Laplace, Scharr operators are shown in figures. When we compare Figure 4(b) with Figure 4(c)-(f) and it can be understood that the level of detection of the edge details accomplished using the proposed algorithm is very much better than the other approaches used in the experiment. In the other approaches shown there is a lot of information unavailable and as a result of which the diagnosis process becomes difficult for the physician. In order to obtain an edge detected image to overcome the issues noticed in Figure 4(c), image fusion is suggested between Sobel 12D and Laplace filter, since the edge detection outcome generated using 
them seems to be better. Figure $4(\mathrm{~g})$ shows the output of image fusion and the resultant image obtained are very much better compared to the other images generated. The edges extracted are found to be continuous and information recovery can be performed without interruption.



(a)

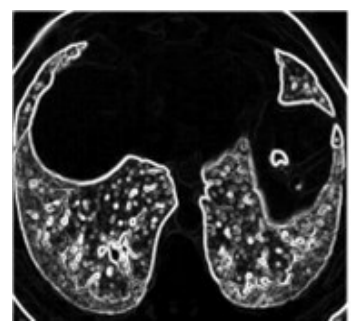

(b)

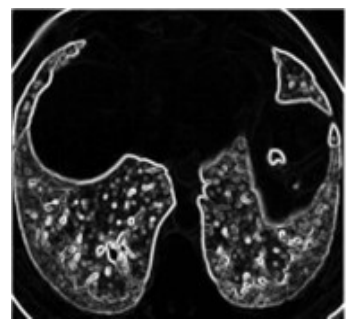

(c)

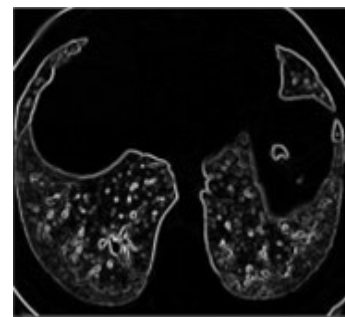

(d)

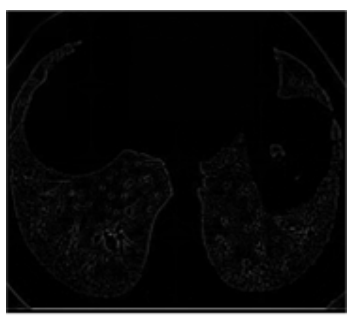

(e)

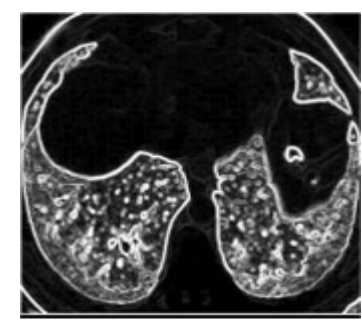

(f)

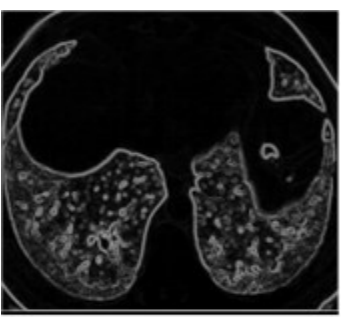

(g)

Figure 4. Traditional operator and modified operator edge image of pulmonary image: (a) original image, (b) Sobel12d operator, (c) Prewitt operator, (d) Roberts operator, (e) Laplace operator, (f) Scharr operator, and $(\mathrm{g})$ image fusion approach

Finally, the optimization of the proposed approach of edge detection is performed using the image fusion approach. According to the algorithm proposed the different directional templates are implemented along with the edge detection process. As a result of which the required edge detected image is generated. This helps the physician diagnose the various parameters to be highlighted in the image's edges. It is also observed that the usage of the different direction templates shows additional information about the original input image. The additional parameters focused is information about the edges and also edges contains more data. The various issues such as problem with lack of smoothness, interval between the edge parts identified in the traditional Sobel edge detection are resolved here. Finally, the edges detected are very much better compared to other methods available. Figure 5 illustrates the enhanced results for the same case of IPF shown in Figure 4 and Figure 5(a) is the original "intralobular interstitial thickening" image. As a part of illustration of the final enhanced pulmonary image there are different results generated using the various existing operators shown in Figures 5(b)-(g). Figure 5(b) shows the enhanced output obtained as a result of the Sobel $12 \mathrm{D}$ algorithm. The performance evaluation of the proposed approaches and the existing approaches are shown in Table 1.

Table 1. Performance metric values for various edge detection operators and proposed approaches

\begin{tabular}{clcccccc}
\hline IPF Image Types & Metric & Laplace & Prewitt & Robert & Scharr & Sobel 12D & Fusion \\
\hline IIT & PSNR & 24.094 & 13.682 & 15.904 & 12.155 & 26.418 & 25.934 \\
& RMSE & 11.266 & 52.771 & 40.862 & 62.918 & 28.486 & 22.828 \\
& SSIM & 0.931 & 0.655 & 0.725 & 0.535 & 0.6333 & 0.847 \\
& EPI & 0.995 & 0.948 & 0.966 & 0.928 & 0.945 & 0.982 \\
& Entropy & 6.56 & 5.93 & 5.87 & 6.23 & 6.88 & 6.86 \\
& PSNR & 23.860 & 14.613 & 17.937 & 12.926 & 24.877 & 23.818 \\
& RMSE & 6.509 & 47.412 & 32.334 & 57.575 & 25.991 & 21.038 \\
& SSIM & 0.962 & 0.5816 & 0.760 & 0.405 & 0.512 & 0.784 \\
& EPI & 0.999 & 0.9739 & 0.985 & 0.965 & 0.968 & 0.984 \\
& Entropy & 6.34 & 6.15 & 6.35 & 6.01 & 6.76 & 6.98 \\
& PSNR & 20.094 & 13.682 & 15.682 & 12.155 & 21.418 & 22.934 \\
& RMSE & 11.266 & 52.771 & 40.862 & 62.918 & 28.486 & 25.828 \\
& SSIM & 0.931 & 0.655 & 0.725 & 0.535 & 0.633 & 0.847 \\
& EPI & 0.995 & 0.982 & 0.966 & 0.928 & 0.945 & 0.982 \\
& Entropy & 5.98 & 5.88 & 6.23 & 6.45 & 6.67 & 6.78 \\
\hline
\end{tabular}




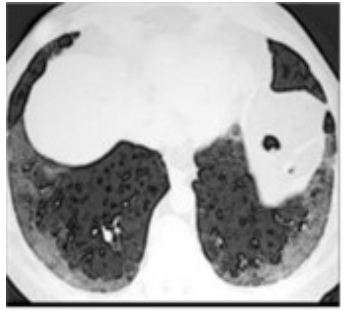

(a)

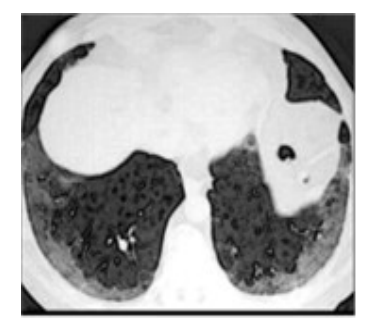

(e)

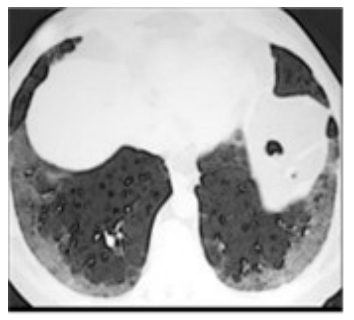

(b)

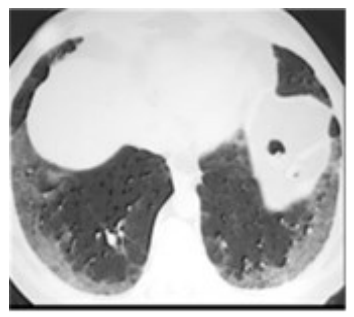

(c)

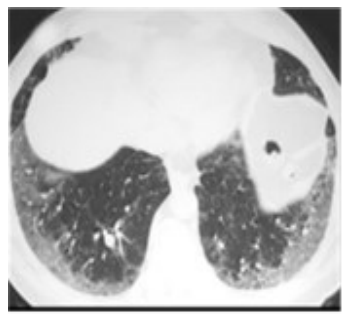

(d)



(f)

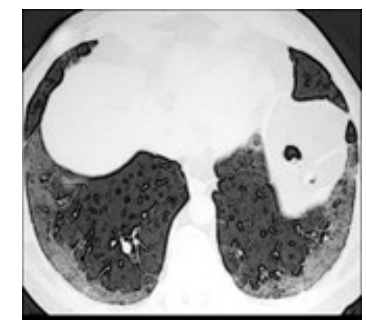

(g)

Figure 5. Enhanced pulmonary output image: (a) original image, (b) Sobel12D operator, (c) Prewitt operator, (d) Roberts operator, (e) Laplace operator, (f) Scharr operator, and (g) image fusion approach

The performance assessment parameters used here are peak signal to noise ratio (PSNR), structured similarity indexing method (SSIM), root mean square error (RMSE), entropy power inequality (EPI) and entropy. The identification of the compatibility of the proposed approaches are performed based on these parameter values calculated. Four cases of IPF were used here for the performance evaluation of the proposed approaches and the existing operators for enhancement. The PSNR value is found to be very much better for the Laplace operator, Sobel 12D and the image fusion approaches. Since a higher value of PSNR denotes the enhancement factor as sharpness to be good. EPI metric used here determines the amount of noise removed and edge preservation achieved.

The other metrics used for evaluation also shows the better edge detection for the proposed approaches compared to the standard operators. Thereby it can be concluded that the algorithm proposed in the work is very much better than the other edge detection techniques available. So the enhanced output image obtained is also found to be much clear and better in the proposed approaches.

\section{CONCLUSION}

This study proposes a modified Sobel edge detection approach along with an image fusion method for better edge detection and enhancement of the CT scan pulmonary images. The diagnosis of the various stages of IPF is performed successfully. Three cases of IPF which focuses on the boundaries are mainly considered for the performance evaluation and most of them shows the superiority of the proposed approaches. This study describes the possible applicability of the proposed technique in medical image diagnostic and detailed understanding of the edges of the CT scan pulmonary images. Although the proposed method enhances color images (as well as grayscale image) the output image generated remains grayscale. This is an immediate future research direction.

\section{REFERENCES}

[1] M. Nikolic, E. Tuba, and M. Tuba, "Edge detection in medical ultrasound images using adjusted Canny edge detection algorithm," in 2016 24th Telecommunications Forum (TELFOR), 2016, pp. 1-4, doi: 10.1109/TELFOR.2016.7818878.

[2] M. Diwakar and M. Kumar, "A review on CT image noise and its denoising," Biomed. Signal Process. Control, vol. 42, pp. 73-88, 2018, doi: 10.1016/j.bspc.2018.01.010.

[3] J. N. Archana and P. Aishwarya, "A Wavelet Based Unsharp Masking Algorithm for Enhancing Lung Images," no. 12, pp. 2597-2602, 2019, doi: 10.35940/ijitee.K2091.1081219.

[4] A. Depeursinge, H. Müller, A. Hidki, P.-A. Poletti, A. Platon, and A. Geissbuhler, "Image-based diagnostic aid for interstitial lung disease with secondary data integration," in Medical Imaging 2007: Computer-Aided Diagnosis, vol. 6514, p. 65143P, 2007, doi: 10.1117/12.709533. 
[5] P. A. Thabsheera, T. M. Thasleema, and R. Rajesh, "Lung cancer detection using CT scan images: A review on various image processing techniques,” Data Anal. Learn., pp. 413-419, 2019, doi: 10.1007/978-981-13-2514-4_34.

[6] P.-W. Hsieh and P.-C. Shao, "Blind image deblurring based on the sparsity of patch minimum information," Pattern Recognit., vol. 109, p. 107597, 2021, doi: 10.1016/j.patcog.2020.107597.

[7] W.-C. Lin and J.-W. Wang, "Edge detection in medical images with quasi high-pass filter based on local statistics," Biomed. Signal Process. Control, vol. 39, pp. 294-302, 2018, doi: 10.1016/j.bspc.2017.08.011.

[8] S. H. Abdulhussain, A. R. Ramli, B. M. Mahmmod, S. A. R. Al-Haddad, and W. A. Jassim, "Image edge detection operators based on orthogonal polynomials," Int. J. Image Data Fusion, vol. 8, no. 3, pp. 293-308, 2017, doi: $10.1080 / 19479832.2017 .1326405$.

[9] S. V. M. Sagheer and S. N. George, "A review on medical image denoising algorithms," Biomed. Signal Process. Control, vol. 61, p. 102036, 2020, doi: 10.1016/j.bspc.2020.102036.

[10] Y. Liu, Z. Xie, and H. Liu, "An adaptive and robust edge detection method based on edge proportion statistics," IEEE Trans. Image Process., vol. 29, pp. 5206-5215, 2020, doi: 10.1109/TIP.2020.2980170.

[11] W. Wong, C. J. Ryerson, and S. A. Guler, "Progression of fibrosing interstitial lung disease," Respir. Res., vol. 21, no. 1, pp. 1-10, 2020, doi: 10.1186/s12931-020-1296-3.

[12] L. Richeldi, H. R. Collard, and M. G. Jones, "Idiopathic pulmonary fibrosis," Lancet, vol. 389, no. 10082, pp. 1941-1952, 2017, doi: 10.1016/S0140-6736(17)30866-8.

[13] D. Bouros, V. Tzilas, and A. Tzouvelekis, "Diagnostic guidelines for IPF: when art meets science," Lancet Respir. Med., vol. 6, no. 11, pp. 812-814, 2018, doi: 10.1016/S2213-2600(18)30430-28.

[14] M. Chhabra and R. Kumar, "Comparison of Different Edge Detection Techniques to Improve Quality of Medical Images," J. Comput. Theor. Nanosci., vol. 17, no. 6, pp. 2496-2507, 2020, doi: 10.1166/jctn.2020.8921.

[15] J. C. Reed, "Chest Radiology: Patterns and Differential Diagnoses," 7e. 2018.

[16] J. Cao, L. Chen, M. Wang, and Y. Tian, "Implementing a parallel image edge detection algorithm based on the Otsu-canny operator on the Hadoop platform," Comput. Intell. Neurosci., vol. 2018, 2018.

[17] R. A. Purba, J. Sembiring, E. H. Sihombing, and S. Sondang, "Edge image detection combines Laplace operation with convolution technique to produce drawing materials for children," in Journal of Physics: Conference Series, vol. 1402, no. 6, 2019, p. 66097, doi: 10.1088/1742-6596/1402/6/066097.

[18] Q. Tian, G. Xie, Y. Wang, and Y. Zhang, "Pedestrian detection based on laplace operator image enhancement algorithm and faster R-CNN," in 2018 11th International Congress on Image and Signal Processing, BioMedical Engineering and Informatics (CISP-BMEI), pp. 1-5, 2018, doi: 10.1109/CISP-BMEI.2018.8633093.

[19] M. Sharifi, M. Fathy, and M. T. Mahmoudi, "A classified and comparative study of edge detection algorithms," in Proceedings. International conference on information technology: Coding and computing, pp. 117-120, 2002.

[20] D. J. Vincent and V. S. Hari, "Edge enhancement and noise smoothening of CT images with anisotropic diffusion filter and unsharp masking," in 2018 IEEE Recent Advances in Intelligent Computational Systems (RAICS), pp. 55-59, 2018, doi: 10.1109/RAICS.2018.8635086.

[21] C.-C. Zhang, J.-D. Fang, and P. Atlantis, "Edge Detection Based on Improved Sobel Operator," in Proceedings of the 2016 International Conference on Computer Engineering and Information Systems, vol. 52, pp. 129-132, 2016, doi: 10.2991/ceis-16.2016.25.

[22] W. Gao, X. Zhang, L. Yang, and H. Liu, "An improved Sobel edge detection," in 2010 3rd International conference on computer science and information technology, vol. 5, pp. 67-71, 2010, doi: 10.1109/ICCSIT.2010.5563693.

[23] J. Naam, J. Harlan, R. Syelly, and A. Ramadhanu, "Filter technique of medical image on multiple morphological gradient (MMG) method," TELKOMNIKA (Telecommunication, Computing, Electronics and Control), vol. 17, no. 3, pp. 1317-1323, 2019, doi: 10.12928/TELKOMNIKA.v17i3.9722.

[24] G. Chen, Z. Jiang, and M. M. Kamruzzaman, "Radar remote sensing image retrieval algorithm based on improved Sobel operator,” J. Vis. Commun. Image Represent., vol. 71, p. 102720, 2020, doi: 10.1016/j.jvcir.2019.102720.

[25] X. Tang, X. Wang, J. Hou, H. Wu, and D. Liu, "An Improved Sobel Face Gray Image Edge Detection Algorithm," in 2020 39th chinese control conference (CCC), 2020, pp. 6639-6643, doi: 10.23919/CCC50068.2020.9189302.

[26] K. Zhang and Q. Liao, "FPGA implementation of eight-direction Sobel edge detection algorithm based on adaptive threshold," in Journal of Physics: Conference Series, vol. 1678, no. 1, 2020, p. 12105, doi: 10.1088/17426596/1678/1/012105.

[27] M. Jing and Y. Du, "Flank angle measurement based on improved Sobel operator," Manuf. Lett., vol. 25, pp. 44-49, 2020, doi: 10.1016/j.mfglet.2020.07.002.

[28] H. H. Abbass and Z. R. Mousa, "A proposed method for face image edge detection using Markov basis," Int. J. Comput. Sci. Inf. Secur., vol. 15, no. 2, p. 470, 2017.

[29] Y. Zhang, X. Han, H. Zhang, and L. Zhao, "Edge detection algorithm of image fusion based on improved Sobel operator," in 2017 IEEE 3rd Information Technology and Mechatronics Engineering Conference (ITOEC), pp. 457-461, 2017, doi: 10.1109/ITOEC.2017.8122336.

[30] L. Han, Y. Tian, and Q. Qi, "Research on edge detection algorithm based on improved Sobel operator," in MATEC Web of Conferences, vol. 309, p. 3031, 2020, doi: 10.1051/matecconf/202030903031. 


\section{BIOGRAPHIES OF AUTHORS}
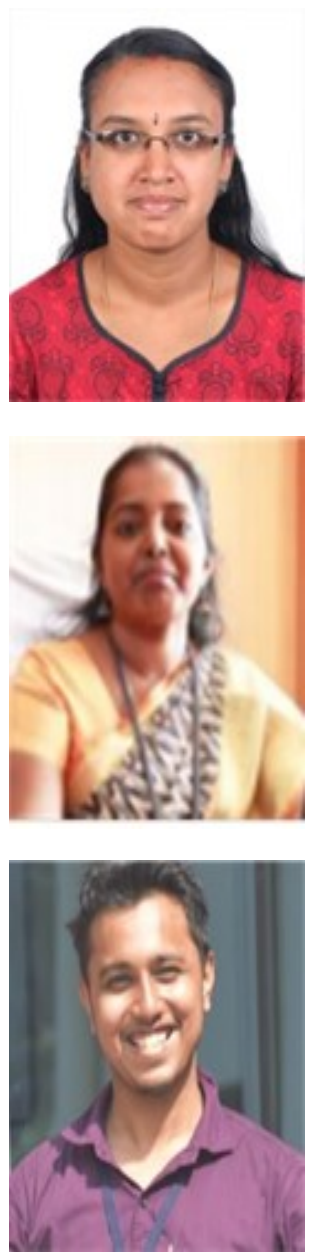

Archana J. N. completed her M. Tech in Computer Science and Engineering from Anna University Chennai and pursuing her Ph. D in Image Processing from Bharathiar University, Coimbatore., India. She has more than 14 Years Teaching Experience and is currently working as a Adhoc Faculty in NIT, Calicut. Her Research area is Image Processing, Networking.

Aishwarya P. completed her Ph.D in Image Processing from M.S University Tirunelveli in 2013. Currently she is the Head of the Department in the CSE Department of Atria Institute of Technology, Bangalore, India. She has more than 18 Years of Teaching Experience. Her Research interests are Image Processing and Networking.

Hanson Joseph is a student of Engineering Physics, Department of Physics from National Institute of Technology, Calicut. His Research interests includes Image Processing, Convolutional Neural Networks, Machine Learning. 